\title{
Aggregation of Nanochemical Microcrystals in Urine Promotes the Formation of Urinary Calculi
}

\author{
Yanting Lou $\left(D,{ }^{1}\right.$ Wei He, ${ }^{1}$ and Zhengyao Song ${ }^{2}$ \\ ${ }^{1}$ Department of Urology, Chaohu Hospital Affiliated to Anhui Medical University, Chaohu 238000, Anhui, China \\ ${ }^{2}$ Department of Urology, First Affiliated Hospital of Anhui Medical University, Hefei 230022, Anhui, China \\ Correspondence should be addressed to Yanting Lou; huliangliang@ahmu.edu.cn
}

Received 6 August 2020; Revised 9 September 2020; Accepted 16 September 2020; Published 5 October 2020

Academic Editor: Tifeng Jiao

Copyright (c) 2020 Yanting Lou et al. This is an open access article distributed under the Creative Commons Attribution License, which permits unrestricted use, distribution, and reproduction in any medium, provided the original work is properly cited.

With the increasing incidence and recurrence rate of urinary calculi, urinary calculi have become a serious health risk, and the research on urinary calculi has become the focus of public attention. At present, the research results on the formation mechanism of urinary calculi are not ideal, and there is no unified conclusion. In order to further study the influencing factors of the formation of urinary calculi and provide new ideas for the prevention and clinical treatment of urinary calculi, the influence of agglomeration of nanochemical microcrystals in urine on urinary calculi was studied in this paper. In this study, fresh morning urine was collected from 10 urological stone patients and 10 healthy controls without urological stone in the urology department of a hospital. After processing the experimental specimens, we first use flame atomic absorption spectrometry and alcian blue colorimetric method to detect the content of $\mathrm{Ca}^{2+}$ and citrate in the urine and then use the nanoparticle size analyzer to detect the microcrystals in the urine. Diameter, distribution, degree of aggregation and potential, and finally HRTEM observation to observe the morphology, chemical composition, and element composition of the nanocrystals. The results showed that the content of $\mathrm{Ca}^{2+}$ and lemon hydrochloric acid in the urine of the experimental group was lower than that of the control group. The particle size of the nanocrystals increased with the increase in the pore size of the membrane. The average particle size of the experimental group increased gradually from $163 \pm 31 \mathrm{~nm}$ to $3219 \pm 863 \mathrm{~nm}$, while the average particle size of the control group increased from $183 \pm 65 \mathrm{~nm}$ to $997 \pm 522 \mathrm{~nm}$. The mean value of the potential decreased with the increase in the pore size of the filter membrane. The change amplitude of the experimental group was $6.57 \mathrm{mV}$, while the change amplitude of the control group was only $1.75 \mathrm{mV}$. In the composition of nanocrystals, element $\mathrm{O}$ accounts for the most, accounting for $42.54 \%$ of all elements. This indicates that the aggregation of nanocrystals in urine will lead to the rapid increase in the size of nanocrystals, which will eventually lead to the formation of stones.

\section{Introduction}

1.1. Background Significance. With the improvement of people's living standard, the economic income and diet structure have changed. The increase in protein and fat intake leads to the gradual decline of people's body function, and the incidence and recurrence rate of urinary calculi gradually increase, becoming one of the common diseases of urology worldwide [1]. Patients with urinary calculi will have severe pain, hematuria, urinary tract infection, renal injury, and other symptoms, which will bring a great impact on the normal life of patients [2]. At present, the research results on the formation mechanism of this disease are few and not conclusive. Therefore, further research on the formation factors of urinary calculi is of great significance for the prevention and treatment of urinary calculi.

1.2. Related Work. Urinary calculi, as a disease with a high incidence and recurrence rate, have become the focus of medical research, and many researchers at home and abroad have investigated it. Habashy et al. studied whether the component analysis of urinary calculi determined by DECT scanning would lead to changes in patient management, performed DECT-KUB examination on all patients with renal colic symptoms who did not receive DECT 
scanning, and reviewed the DECT data of all patients from September 2013 to July 2015 [3]. Their data, though extensive, are laborious to work with. Turk et al. summarise the latest recommendations of the Urological Guidelines Group of the European Urological Association for the interventional treatment of ureters and kidney stones [4]. Sewell et al. introduced the evidence-based guidelines from diagnosis to conservative surgical treatment and prevention of urinary calculi [5]. Both of their studies are limited to theoretical analysis and do not carry out corresponding experiments. Gkentzis A analyzed the pathogenesis and treatment of urolithiasis combined with inflammatory bowel disease (IBD) and the relevant literature after bariatric surgery. PubMed was used for systematic literature retrieval and other references to evaluate the correlation between IBD bariatric surgery and renal calculi in children and adults [6]. Some of the references they analyzed were old enough to be of little significance for the present situation.

1.3. Innovative Points in This Paper. In order to study the relationship between the agglomeration of nano-chemical microcrystals in urine and the formation of urinary calculi, and to provide new ideas for the prevention and treatment of urinary calculi, this study was conducted on fresh morning urine from urinary calculi patients and healthy controls in the urology department of a hospital For collection and processing, the experimental group $(n=10)$ is the urine of patients with urinary calculi, and the control group $(n=10)$ is the urine of the healthy control group. By detecting the contents of citrate and $\mathrm{Ca}^{2+}$ in urine, the particle size, distribution, aggregation degree, and $\xi$ potential of the nanocrystals, as well as the morphology, chemical composition, and elemental composition of the nanocrystals, the conclusion was finally reached that the agglomeration of the nanocrystals in urine promoted the increase in the particle size of the nanocrystals, which eventually led to the formation of stones.

\section{Nanocrystallite Agglomeration and Urinary Calculi}

\subsection{Urological Stone Formation}

2.1.1. Formation Mechanism of Urinary Calculi. The supersaturated crystallization theory believes that the stoneforming components in the urine are saturated enough and precipitated to form crystal nuclei, which grow into small particles with higher free energy through solute transport and surface action. After agglomeration under some physical and chemical forces, they are collected in the collecting tube or stay in the urinary tract, and may continue to grow and accumulate, eventually forming urinary stones [7]. Among them, supersaturation is the chemical driving force for the formation of urinary calculi because it is a necessary condition for the formation of crystal nucleus. The retention of crystals is another important cause of stone formation. When crystals remain in the urinary tract, they will grow and accumulate, leading to stones. However, in fact, the crystal components in the urine of normal people are often in a supersaturated state, but not everyone can form urinary stones, because the supersaturated crystal theory ignores the organic molecules and other molecules in the urine and only focuses on the role of inorganic salts.

According to the Randall plaque theory, calculi originated from the calcium salt deposition at the renal papillary area, and Randall plaque originated from the basement membrane of the fine segment of Henle's capillary, and gradually developed from the stroma to the urinary epithelium. When plaque broke through the urinary epithelium and was exposed to urine, it became the origin of urinary calculi $[8,9]$. Moreover, the collagen bundles between the renal papilla and the basement membrane can promote the adsorption and deposition of inorganic salts. The Randall plaque theory cannot fully explain the formation of urinary calculi, but it is of great significance for the study of the formation of urinary calculi.

The theory of inhibitor deficiency holds that there is a certain amount of substances in urine that can inhibit crystallization nucleation or growth aggregation, which can inhibit the formation of urinary calculi, and the lack of such substances may lead to calculi. Many substances have been found to inhibit the formation of stones, such as citric acid, renocalcin, ribonucleic acid, Tamm-Horsfall protein, and glucan [10]. They can be combined with crystal growth points, block crystallization nucleation, growth, and aggregation and can also be combined with some stone components in urine to form chelates to reduce saturation. At present, potassium citrate and orthophosphate and other drugs have been used clinically to treat or prevent urinary calculi [11].

The stroma theory believes that stroma is an activator to promote the formation of urinary calculi. It can combine with apatite to form small particles, which can induce the nucleation of calculi components in the suprasaturated urine and provide a template for calculi growth. Acidic mucosaccharides, carbohydrates, and proteins are the main components of the matrix, and the weight of the matrix can account for about $5 \%$ in stones [12].

2.1.2. Etiology of Urinary Calculi. Obesity is an important factor affecting urinary calculi. Obese people consume more energy and have a lower $\mathrm{PH}$ value in urine, thus reducing the excretion of citric acid and increasing the excretion of oxalic acid [13]. In addition, the unbalanced diet of obese people will make them consume too much meat and fat food and lack dietary fiber, which is more likely to lead to the formation of urinary stones.

Diabetes can increase the risk of urinary stones. Compared with the general population, diabetic patients have a lower $\mathrm{pH}$ value of urine and a higher solubility of urinary calcium. Glucose and uric acid in urine will play a competitive role in reabsorption [14]. The increased excretion of oxalate and uric acid leads to the formation of calculi, which is also the reason why diabetic patients are prone to oxalate calculi and urate calculi. 
Hyperlipidemia was positively associated with the formation and recurrence of urinary calculi. High lipid and protein diets promote the increase in lipid in renal tubules, weaken the function of sodium-hydrogen ion exchangers, and acidify urine, leading to the formation of calculi. Moreover, hyperlipidemia will cause inflammation. When inflammatory mediators gather in the kidney, renal mucosa will be easily damaged, creating conditions for the formation of stones.

Abnormal metabolism is closely related to the formation of urinary calculi, including abnormal oxalic acid metabolism, abnormal purine metabolism, abnormal citrate metabolism, abnormal cystinuria, and abnormal calcium metabolism, which will increase the risk of urinary calculi.

\subsubsection{Diagnosis and Treatment of Urinary Calculi. In ad-} dition to clinical diagnosis and laboratory examination, the final diagnosis of urinary calculi requires imaging examination. The main examination methods include ultrasound, $\mathrm{X}$-ray, CT, and intravenous urography (IVU). Among them, the most widely used examination means are ultrasound image and X-ray plain film. Most of the stones with a particle size greater than $0.2 \mathrm{~cm}$ can be found by ultrasound image, and whether the urinary tract at the proximal end of the stones is dilated can be observed [15]. X-ray plain film can visually observe the size, location, number, and shape of calculi, but there are some limitations in the examination of uric acid calculi, with low sensitivity. After IVU was injected intravenously with contrast agent, the development of contrast agent in the urinary tract was used for dynamic $\mathrm{X}$-ray radiograph observation, which could not only observe the stones but also understand the relevant conditions of the kidney. CT can find small stones, is easy to operate quickly, and has good sensitivity and specificity.

The treatment of urinary calculi is divided into two categories: conservative treatment and surgical treatment.

Conservative treatment uses drugs for stone expulsion, which must meet the following requirements: diameter less than $6 \mathrm{~mm}$ or so, smooth surface, local residence time less than two weeks, and no obvious obstruction in the lower ureter [16]. Although some minimally invasive stone therapies can be effective in treating small and small stones, these techniques are risky and expensive. Patients without infection can choose to observe and wait appropriately and take oral medication to control pain, but this is closely related to pain tolerance and uncertainty and the potential risk of renal dysfunction. When using drugs to remove stones, attention should be paid to maintain adequate amount of water and appropriate activities every day, which can help to remove stones. At present, the commonly used drugs for the treatment of urolithiasis include nonsteroidal anti-inflammatory drugs, opioid analgesics, calcium ion blockers, and spasmolytic drugs.

Surgical treatment included extracorporeal shock wave therapy, ureteroscopic lithotripsy, percutaneous nephrolithotripsy, open surgery, and laparoscopic surgery. Extracorporeal shock wave therapy uses the high-energy shock wave generated by the lithotriptor to break the calculi in the body. However, in order to avoid the damage to the kidney caused by the shock wave, the frequency of each operation shock wave is not more than 5 times, and the interval of each operation is at least half a month. Ureteroscopic lithotripsy was performed to treat retrograde calculus through ureteroscopy or ureteral soft view, and calculus was shattered with holmium laser. Percutaneous nephrolithotripsy uses puncture technology to reveal the establishment of percutaneous renal channels and uses pneumatic trajectory and ultrasound to break stones. However, the selection of puncture sites is not easy and often leads to complications such as bleeding and infection. Open surgery mainly involves the lithotomy of kidney, ureter, and bladder, and its application has been significantly reduced.

\subsection{Growth Agglomeration Theory of Chemical Nanocrystals}

2.2.1. Crystal Growth Theory. Crystal growth is not the combination of small particles but the result of crystal interface movement. The velocity of interfacial movement is related to the concentration difference between the surface layer and the inner layer, the curvature of the interfacial layer, and the temperature of the system. The higher the temperature is, the smaller the curvature radius is, and the faster the crystal interface moves to the center of curvature. Whether the pores move with or against the crystal interface is related to the curvature of the crystal interface and the diameter, number, diffusion rate, and gas pressure of the pores [17]. Another reason for limiting crystal growth is the presence of a small amount of liquid at the grain boundary. Due to a small amount of liquid phase, two new solid-liquid interfaces are formed at the grain boundary. The advancing force of interface movement decreases, and the diffusion distance becomes longer. Therefore, a small amount of liquid phase may inhibit crystal growth.

According to the traditional crystallization theory, there is a nearly static layer on the side near the solid, which is similar to the effect of a liquid film. This layer of liquid film can increase the solute diffusion resistance, and the size of the resistance is related to the thickness of the liquid film, temperature, and other factors.

The crystal interface has the effect of interfacial energy, and the grains will form a 3D array. If the surface tension of the crystal interface is the same, the grains will show a regular hexagon [18]. If the second kind of inclusions at the crystalline interface cannot be thought of as liquid phase at the sintering temperature, they will love the movement of the crystalline interface.

2.2.2. Surface Dynamics of Nanocrystalline Growth. The main reason for the growth of nanocrystals is the presence of the mixture film, which consists of solvent and water. In the process of growing nanocrystals, liquid film exists in the solid-gas system, while in the general process of crystal growth, liquid film exists in the solid-liquid system [19]. Due to the presence of solvents in the liquid film, the concentration difference on both sides of the solid and liquid film will occur, which is the main driving force for the growth of 
nanocrystals. When the temperature increases, the thickness of liquid film increases and the viscosity decreases, and the growth of nanocrystals will be accelerated.

On the basis of diffusion theory, the formula for calculating the surface chemical kinetics of microcrystalline growth is shown:

$$
D=\frac{M \delta N\left(Q_{1}-Q_{2}\right)}{\eta},
$$

where $M$ is the specific surface area, $\delta$ is the thickness of the liquid film, $N$ is the temperature, $Q_{1}$ and $Q_{2}$ are the concentrations of the liquid film on both sides of the gas phase and the liquid phase, respectively, and $\eta$ is the viscosity of the liquid film.

At constant temperature and constant pressure, a certain volume of crystal forms an equilibrium shape, at which time the crystal surface energy is minimum. The equilibrium morphology is determined by the normal growth rate of each crystal, and the normal growth rate is proportional to the specific surface freedom. If the specific surface of the mirror changes freely, the equilibrium form will also change accordingly.

2.2.3. Nanocrystalline Agglomeration Mechanism. The kinetic reason for agglomeration is that, under a certain environment, crystal particles are affected by gravity and the interaction force. However, when the interaction force is greater than gravity, the particles are constrained by gravity to a lower degree, resulting in the occurrence of microcrystal agglomeration [20]. The main interactions between microcrystalline particles include van der Waals force and Coulomb force.

The criterion of microcrystalline agglomeration is the ratio of the force causing agglomeration to gravity, and its expression formula is shown:

$$
P=\frac{D}{m g},
$$

where $D$ is the sum of all forces causing agglomeration of microcrystals and $m g$ is the gravity.

In addition to the influence of force, the morphology of microcrystal also affects the agglomeration of microcrystals to a certain extent. The morphology of microcrystalline is closely related to the surface energy, and the stable morphology of microcrystalline must have the minimum surface energy. Therefore, the growth of microcrystalline agglomerations, the decrease in specific surface area, and the tendency of particle surface energy to the minimum are all spontaneous thermodynamic processes. The basic way to solve micrite accumulation is to reduce the surface free energy of each crystal surface.

2.3. Methods of Urine Detection. Urine detection includes the detection of physical properties, chemical composition, and microparticles, and the detection of microparticles in urine is required in the detection of urinary calculi. The detection methods of microparticle spatial distribution in urine include flow cytometry, microimage morphological analysis, and spatial dynamic coordinate tracking detection.

2.3.1. Flow Cytometry. Flow cytometry first requires fluorescent pigment staining of the tested specimens, and then, it is put into the sample tube to make it enter the flow chamber under the action of gas pressure. The flow chamber is full of hydrosheath, which can restrain the cells into a single row and form a cell column when it is ejected from the nozzle [21]. The fluorescence of the cells can be detected by the instrument to obtain relevant information. Through the calculation and analysis of the information, the size, length, volume, and chromatin length of the cells can be determined. Finally, according to the morphological characteristics of the cells, the scattering diagram and related reports of urine microparticles can be formed.

Flow cytometry is generally used in blood testing. The optical system in the device can provide fluorescence, light scattering, light absorption, and other signals, while the computer system can provide signal acquisition, storage, display, and analysis services, which can be used for rapid and automatic detection and statistical analysis of various indicators [22].

However, this method cannot detect all the microscopic particles, and when the urine samples contain luciferin or larger particulate pollutants, the reliability will be reduced and the diversion tube will be blocked. If the concentration of urine sample is too high, it will affect the results of the next sample, and this test cannot obtain a complete microscopic image of urine nor can it correct the results. The detection does not have traceability nor can it completely avoid the situation of less inspection and even wrong inspection.

2.3.2. Morphological Analysis of Microscopic Images. Morphological analysis of microscopic image obtains digital image of microscopic particles of urine by imaging equipment, and the results are obtained after processing and analyzing the digital image. The urine microparticle image detection method is an intuitive observation method. Before the image is obtained in the imaging system, the samples can be centrifuged at a certain speed and time, and the samples separated by centrifugation can be transferred to the cargo platform of the electron microscope [23]. Based on the microscopic particle images obtained by the imaging system, the next operation is carried out.

With the development of the automatic urine microparticle analysis system, the image-based urine microparticle analysis device has gradually developed from single functions such as image acquisition, patient information management, and examination report generation to automatic sampling collection and automatic focusing, image processing, particle recognition, counting, and reporting functions are integrated multifunctional detection and analysis system.

Morphological analysis of microscopic images has the advantages of traceability, visualization, meeting the gold standard of microscopy, drawing lessons from doctors' 
experience, and reporting particle openness, but it is an important technology that needs to improve detection methods and technical means [24], for example, effective detection methods, high-quality image acquisition methods, subsequent image processing, segmentation, pattern recognition, algorithm effectiveness, and accuracy.

At present, most of the equipment detection system and detection item report are based on the statistical principle and normal distribution principle. Compared with the blood test, the urine test has certain specificity because of its complex granules and short specimen time.

\subsubsection{Spatial Dynamic Coordinate Tracking Detection} Method. At present, the general detection method requires the natural sedimentation of urine. All the urine microscopic particles are deposited at the bottom of the counting tank within a limited time. The microscopic particles in the detection tank can be photographed by the mobile platform and then analyzed [25]. This method is simple and convenient, but it has its drawbacks. For example, the settlement effect is not ideal, and all the microscopic particles cannot be guaranteed to settle to the bottom; the settlement time is long, and the sufficient settlement cannot be achieved within a short time and cannot avoid missing or less inspection.

Therefore, the spatial dynamic coordinate tracking and monitoring method of surface settlement or short-time settlement can be adopted. Therefore, the spatial dynamic coordinate tracking monitoring method of surface settlement or short-term settlement can be used for analysis. When the microscopic particles are not sufficiently settled, the focal plane needs to be focused on multiple levels from top to bottom, and the spatially distributed microscopic particles are decomposed into multilayer planes, and the visible area of the microscope is used for observation to ensure that the suspension can be detected. All the microscopic particles in the upper part of the detection tank can avoid missed and under-detected situations and improve the detection speed.

\section{Experiments on Detection of Nanochemical Microcrystals in Urinary Calculi}

\subsection{Research Objects and Materials}

3.1.1. Research Objects. Ten patients with urinary calculi and ten healthy controls without urinary calculi in the urology department of a hospital were the subjects of this study. Patients with calculi were in the experimental group $(n=10)$, and healthy controls were in the control group $(n=10)$. Their fresh morning urine was collected as experimental specimens. Among them, there were 12 males and 8 females, ranging from 30 to 60 years old. Among them, the largest number was 41-50-year-old, followed by 51-60-year-old, and the least number was under 40 -year-old. After the test, $P>0.05$ was found, and there was no difference in age distribution between the patients and the control group of different genders, as shown in Table 1.
TABLE 1: General information of experimental subjects.

\begin{tabular}{lccc}
\hline Age range & Man & Woman & Total \\
\hline $30-40$ & 3 & 1 & 4 \\
$41-50$ & 6 & 3 & 9 \\
$51-60$ & 3 & 4 & 7 \\
Total & 12 & 8 & 20 \\
\hline
\end{tabular}

Note. $X^{2}=0.581 ; P=0.613$.

3.1.2. Reagents and Instruments. Conventional chemical reagents were analytically pure, and all glassware was cleaned with secondary distilled water.

The instruments include XL-30 environmental scanning electron microscope, Zetasizer Nano-ZS nanometer, X-ray powder diffraction, and KQ3200 DE ultrasonic instrument.

3.2. Collection and Treatment of Urine. Fasting morning urine was collected from 10 patients with urinary calculi and 10 healthy controls. In order to avoid the influence of tissue fragments and other macromolecular particles in urine on the detection results, $40 \mathrm{ml}$ urine samples were collected from the control group and the experimental group, and $20 \mathrm{ml}$ ethanol was added. After stirring for $3 \mathrm{~min}$, the urine samples were placed for $30 \mathrm{~min}$ to remove the proteins. Stir in $15 \mathrm{ml}$ of secondary distilled water dilution, with $0.23,0.5$, $1.5,3.5$, and 10 reserve $\mu \mathrm{m}$ aperture of organic membrane filtration.

3.3. Determination of Citrate and $\mathrm{Ca}^{2+}$ in Urine. The content of $\mathrm{Ca}^{2+}$ in the urine of 10 stone patients and 10 healthy controls was determined by flame atomic absorption spectrometry. The instrument radiates light with $\mathrm{Ca}^{2+}$ element characteristic spectral line from the light source, which is absorbed by $\mathrm{Ca}^{2+}$ ground state atoms in the urine sample when passing through the vapor, and the content of $\mathrm{Ca}^{2+}$ in the urine sample is determined by the degree to which the radiation characteristic spectral line light is weakened.

The citrate content in urine was determined by Alcian blue colorimetry in 10 patients with calculi and 10 healthy controls. Two sets of spare urine samples were placed in test tubes with borax solution with a concentration of $6 \%$ and a $\mathrm{pH}$ of $0.025 \mathrm{~mol} / \mathrm{LpH}$ of 9.0 as electrode buffer. The voltage was adjusted to $100 \mathrm{~V}$, the current was about $3 \mathrm{~mA}$, the electrophoresis lasted for 4.5 hours, and the staining was performed after the electrophoresis. The polyacrylamide gel was fixed in the base solution for 1 hour, oxidized in the oxidation solution for 1.5 hours, reduced for 0.5 hours, soaked in Alcian blue staining solution for more than 2 hours, and finally placed in the base solution until the background was colorless, and then, the citrate content could be detected.

\subsection{Nanocrystalline Detection}

3.4.1. Nanometer Detection. After the two groups of filtered urine were treated with ultrasonic instrument for $5 \mathrm{~min}$, 
the possible agglomeration of urine microcrystals was destroyed and detected by nanometer size analyzer, respectively. The particle size, distribution, aggregation degree, and potential of urine microcrystals could be obtained. The detection time immediately after ultrasound was recorded as $0 \mathrm{~h}$, and the detection time was once every 1 hour, and the detection time was consecutively 3 times.

3.4.2. HRTEM Observation of Urine Microcrystals. After ultrasonic treatment for $3 \mathrm{~min}$, the spare urine was dropped on the surface of the copper mesh. After vacuum drying, it was tested for 2 days to observe the morphology, chemical composition, and element composition of the nanocrystals.

\section{Discussion on Relationship between Agglomeration of Nanochemical Microcrystals and Urinary Calculi}

4.1. Levels of $\mathrm{Ca}^{2+}$ and Citrate in Urine. Urine $\mathrm{Ca}^{2+}$ and citrate concentrations were measured in 10 stone patients and 10 healthy controls, and the results are shown in Table 2.

As shown in Table 2, the content of $\mathrm{Ca}^{2+}$ and citrate in urine of patients with calculus in the experimental group was significantly different from that of healthy controls $(P<0.05)$. The amount of $\mathrm{Ca}^{2+}$ in the urine of the experimental group was lower than that of the control group, but the error value was large. The content of lemon hydrochloric acid in the urine of the control group was higher than that of the experimental group.

\subsection{Nanocrystalline Detection}

4.2.1. Particle Size Comparison. The urine samples of the control group and the experimental group were filtered by organic membranes with different pore sizes of $0.23,0.5,1.5$, 3.5 , and $10 \mu \mathrm{m}$, and then tested with a nanoparticle size analyzer. The average particle size of the nanocrystals in the urine is as follows.

As shown in Figure 1, as the pore size of the organic film keeps increasing, the average particle size of the two groups of urine nanocrystals also keeps increasing. The average diameter of urine nanocrystals in 10 patients with calculi gradually increased from $163 \pm 31 \mathrm{~nm}$ to $3219 \pm 863 \mathrm{~nm}$. In healthy controls, the mean diameter of urine nanocrystal increased from $183 \pm 65 \mathrm{~nm}$ to $997 \pm 522 \mathrm{~nm}$. This indicates that although the particle size of the nanocrystalline increases with the increase in the pore size of the organic film, it is smaller than the corresponding pore size. In addition, the particle size of the nanocrystals in the control group was larger than that in the experimental group at the beginning, but with the increase in pore size, the particle size of the control group was significantly smaller than that of the experimental group.

4.2.2. Distribution and Agglomeration. The distribution and aggregation degree of urine nanocrystals in the experimental group and the control group could be obtained after
TABle 2: Contents of $\mathrm{Ca}^{2+}$ and citrate in urine of the two groups.

\begin{tabular}{lcc}
\hline Group & $\mathrm{Ca}^{2+} /\left(\mathrm{mmol} * \mathrm{~L}^{-1}\right)$ & Citrate $/\left(\mathrm{mg} * \mathrm{~L}^{-1}\right)$ \\
\hline Experimental group & $2.14 \pm 0.83$ & $152 \pm 77.5$ \\
Control group & $2.59 \pm 0.21$ & $226 \pm 113.6$ \\
$P$ & $<0.05$ & $<0.05$ \\
\hline
\end{tabular}

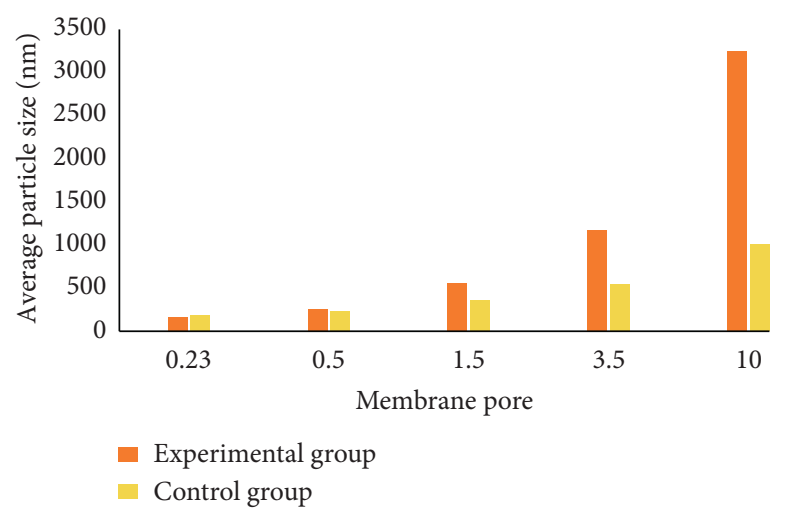

FIGURE 1: Mean particle size changes in urine nanocrystals filtered by organic membranes with different pore sizes.

continuous urine nanoparticle detection. Since there was little change in the control group, the aggregation state of the experimental group was only transformed into a schematic diagram for convenient observation, as shown below.

As shown in Figure 2, there was a small amount of aggregation after ultrasound, almost in a dispersed state. After 1 hour of placement, there was a slight phenomenon of aggregation, but it was mainly composed of a small number of nanocrystals less than $120 \mathrm{~nm}$, with a relatively loose structure. After 2 hours, the degree of aggregation increased significantly, and a larger volume of aggregation was formed. Therefore, it can be proved that the formation of aggregates is the main reason for the rapid increase in the size of nanocrystals. The growth and aggregation of nanocrystals will eventually lead to the formation of stones.

4.2.3. $\xi$ Potential Difference. The $\xi$ potential reflects the electrostatic repulsion between the nanocrystals, and the greater the absolute value of $\xi$ potential corresponds to the greater electrostatic repulsion, which also means that the agglomeration of nanocrystals becomes more difficult. $\xi$ potential in urine is closely related to the ionic concentration of charge and opposite charge on the surface of urine microcrystalline. The urine of the experimental group and the control group was detected by nanometer, and the changes in $\xi$ potential under organic membrane filtration with different pore sizes were recorded. The specific situation was as follows.

As shown in Figure 3, with the continuous increase in the pore size of the organic film, the average value of $\xi$ potential decreases continuously. The mean value of $\xi$ potential in the experimental group decreased from $-2.62 \pm 0.52$ to $-9.19 \pm 2.03$, and the mean value of $\xi$ potential in the control 


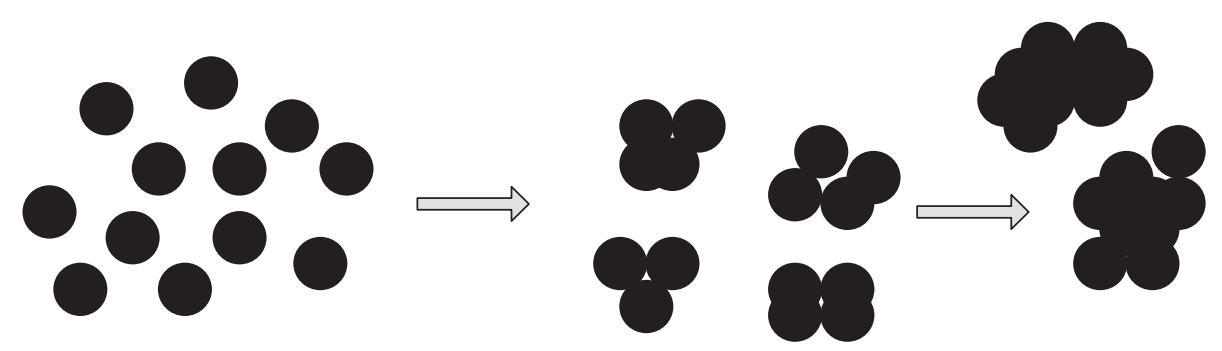

FIGURE 2: Changes of urine microcrystalline aggregates in the experimental group.

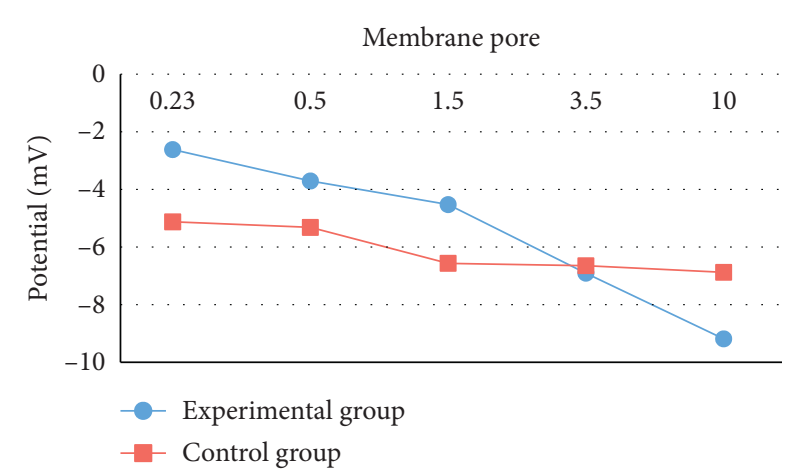

FIGURE 3: Changes in urine microcrystal $\xi$ potential in the two groups.

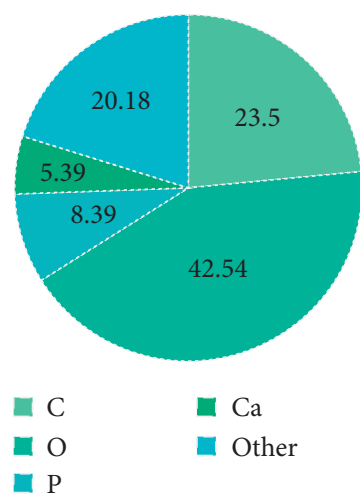

FIGURE 4: Element composition of urine nanocrystalline in a representative patient.

group decreased from $-5.13 \pm 0.93$ to $-6.88 \pm 1.1$. It was obvious that there was a significant difference in the range of change between the two groups. The range of change in the experimental group was $6.57 \mathrm{mV}$, while that in the control group was only $1.75 \mathrm{mv}$.

\subsection{Chemical Element Composition of Nanocrystalline.} HRTEM was used to observe the urine nanocrystals of the two groups of patients, and the chemical elements in the urine nanocrystals of representative stone patients in the experimental group could be detected, mainly four elements $\mathrm{C}, \mathrm{O}, \mathrm{P}$, and $\mathrm{Ca}$. The proportions of these elements are as follows.

As shown in Figure 4, the chemical components and their proportions were accurately detected in the urine nanocrystalline of the patient with the stone. Other elements are temporarily ignored. Among the four main elements C,
$\mathrm{O}, \mathrm{P}$, and $\mathrm{Ca}$, $\mathrm{O}$ element is the most, accounting for $42.54 \%$. The next most important element is element $\mathrm{C}$, accounting for $23.5 \%$. P element and Ca element are second, accounting for $8.39 \%$ and $5.39 \%$, respectively.

\section{Conclusions}

Urinary calculi are common multiple diseases of the urinary system, which can cause urinary tract infection and even lead to renal necrosis in severe cases. At present, the formation mechanism of urinary calculi mainly includes Randall spot theory, saturated crystal theory, inhibitor deficiency theory, and matrix theory. In fact, the formation of urinary calculi is very complex, and there is no unified conclusion on its specific pathogenesis.

The presence of nanochemical microcrystals in urine is closely related to the formation of urinary calculi. Nanocrystals in urine from patients with urinary calculi and healthy controls differed in size, distribution, $\xi$ potential, and composition. The size of urine nanochemical microcrystals in patients with calculi was larger than that in healthy controls, and the degree of aggregation was also higher; urinary calculi were more likely to form.

Due to the limited time and knowledge, the urine detection in this study was not comprehensive enough, and there was no difference in the morphology and light intensity autocorrelation function of urine nanocrystals between stone patients and healthy controls. The above deficiencies will be improved in the next experiment in order to provide more reliable experimental data for the clinical treatment of urinary calculi.

\section{Data Availability}

No data are available.

\section{Disclosure}

Yanting Lou and Wei He are the co-first authors.

\section{Conflicts of Interest}

The authors declare that there are no conflicts regarding this submission.

\section{References}

[1] K. Taguchi, D. S. T. Yasui, and T. ChiHoppe, "Genetic risk factors for idiopathic urolithiasis: a systematic review of the 
literature and causal network analysis," European Urology Focus, vol. 3, no. 1, pp. 72-81, 2017.

[2] B. H. Hancock, J. A. Warren, C. Marguet et al., "Concurrent laparoscopic hernia repair and cystoscopic laser cystolitholaxapy for urinary bladder calculus contained within a direct inguinal hernia," The American Surgeon, vol. 83, no. 9, pp. e406-e408, 2017.

[3] D. Habashy, W. R. Xia, and L. L. RidleyChan, "Impact of dual energy characterization of urinary calculus on management," Journal of Medical Imaging and Radiation Oncology, vol. 60, no. 5, pp. 624-631, 2016.

[4] C. Türk, A. Petř́ik, K. Sarica et al., "EAU guidelines on interventional treatment for urolithiasis," European Urology, vol. 69, no. 3, pp. 475-482, 2016.

[5] J. Sewell, D. J. Katz, O. Shoshany et al., "Urolithiasis-ten things every general practitioner should know," Australian Family Physician, vol. 46, no. 9, pp. 648-652, 2017.

[6] A. Gkentzis, J. M. Kimuli, and C. S. O. BiyaniTraxer, "Urolithiasis in inflammatory bowel disease and bariatric surgery," World Journal of Nephrology, vol. 5, no. 6, pp. 538-546, 2016.

[7] M. B. Carnahan, P. Hoang, and M. Yang, "Demonstration of $99 \mathrm{mTc}-\mathrm{MDP}$ uptake in a mobile urinary bladder calculus on SPECT/CT," Clinical Nuclear Medicine, vol. 45, no. 1, pp. 60-62, 2020.

[8] D. Biswas and A. Saifuddin, "Death of non-descriptive male calf due to urolithiasis followed by rupture of urinary bladder," Bangladesh Journal of Veterinary Medicine, vol. 13, no. 2, p. 63, 2016.

[9] C. Türk, A. Petík, K. Sarica et al., "EAU guidelines on diagnosis and conservative management of urolithiasis," $\mathrm{Eu}$ ropean Urology, vol. 69, no. 4, 2016.

[10] H. Taskinlar, D. D. Yigit, and A. Nayci, "Unusual complication of a urinary stone in a child: spontaneous rupture of the renal pelvis with the migration of calculus into the retroperitoneum," Türk Üroloji Dergisi/Turkish Journal of Urology, vol. 42, no. 1, p. 48, 2016.

[11] J. W. Bartges, "Feline calcium oxalate urolithiasis," Journal of Feline Medicine and Surgery, vol. 18, no. 9, pp. 712-722, 2016.

[12] G. Bailey, C. L. Vaughan, and A. Krambeck, "Perinatal outcomes with tamsulosin therapy for symptomatic urolithiasis," Journal of Urology, vol. 195, no. 1, pp. 99-103, 2016.

[13] M. Bultitude, K. D. Smith, and K. Thomas, "Contemporary management of stone disease: the new EAU urolithiasis guidelines for 2015," European Urology, vol. 69, no. 3, pp. 483-484, 2016.

[14] Y. Yang, Y. Y. Deng, and Y. Wang, "Major geogenic factors controlling geographical clustering of urolithiasis in China," Science of The Total Environment, vol. 571, pp. 1164-1171, 2016.

[15] R. K. Muthusamy and S. S. Mehta, "Eosinophilic pyeloureteritis mimicking urinary calculus: a rare cause of ureteric obstruction in an adult[J]," Open Jounal of Clinical and Medical Case Reports, vol. 2, no. 22, pp. 1-5, 2016.

[16] B. K. Somani, G. Giusti, Y. Sun et al., "Complications associated with ureterorenoscopy (URS) related to treatment of urolithiasis: the clinical research office of endourological society URS global study," World Journal of Urology, vol. 35, no. 4, pp. 1-7, 2016.

[17] E. M. Huseynov, "Investigation of the agglomeration and amorphous transformation effects of neutron irradiation on the nanocrystalline silicon carbide (3C-SiC) using TEM and SEM methods," Physica B: Condensed Matter, vol. 510, pp. 99-103, 2017.
[18] Y. Cao, L. Luo, Q. Guo et al., "Synthesis and agglomeration control of nanocrystalline iowaite," Cailiao Kexue Yu Gongyi/ Material Ence and Technology, vol. 26, no. 2, pp. 27-33, 2018.

[19] E. Magiorkinis, A. Diamantis, and L. Pantanowitz, "The fascinating story of urine examination: from uroscopy to the era of microscopy and beyond," Diagnostic Cytopathology, vol. 43, no. 12, pp. 1020-1036, 2016.

[20] C. Cavanaugh and M. A. Perazella, "Urine sediment examination in the diagnosis and management of kidney disease: core curriculum 2019," American Journal of Kidney Diseases, vol. 73, no. 2, pp. 258-272, 2019.

[21] P. X. Yu, Q. Zhao, Z. M. Meng et al., "Rhabditis axei found in urine routine examination: a case report," Chinese Journal of Schistosomiasis Control, vol. 31, no. 5, pp. 565-566, 2019.

[22] L. Yixiong, T. Zhihong, Y. Meng et al., "Object detection based on deep learning for urine sediment examination," Biocybernetics and Biomedical Engineering, vol. 38, no. 3, pp. 661-670, 2018.

[23] R. Prevel, A. M. Kostine, and M. Souayed, "Dark urine, hypotension and blood smear examination," Intensive Care Medicine, vol. 45, no. 7, pp. 1015-1016, 2019.

[24] K. Dantey, L. Pantanowit, J. Xing, J. Cuda, R. Nestler, and S. E. Monaco, "Cell block preparation in urine cytology: examination of utility and workflow in an academic practice," Journal of the American Society of Cytopathology, vol. 8, no. 2, pp. 61-68, 2019.

[25] L. Jin and C. Cuiling, "Countermeasures and effects of quality control before routine examination and analysis of clinical urine," Journal of Clinical Laboratory (Electronic Edition), vol. 8, no. 1, pp. 72-73, 2019. 